\title{
Evaluation de la technique DEFT associée à un comptage visuel ou à un comptage par analyseur d'images pour la numération de la flore totale du lait cru
}

\author{
A. DASEN *, Christine PITON *, R. GRAPPIN * et Pascale GUERRY ** \\ * I.N.R.A., Station expérimentale laitière, B.P. $n^{\circ}$ 94, 39800 Poligny, France \\ ** Elève ingénieur, I.N.A., Paris-Grignon, France
}

\section{Résumé}

La technique DEFT (Direct Epifluorescent Filter Technique) est une méthode rapide de dénombrement des bactéries du lait cru qui utilise la filtration sur membrane et la microscopie en épifluorescence.

Cent soixante-sept échantillons de lait cru contenant entre $7.10^{3}$ et $4.10^{6} \mathrm{UFC} / \mathrm{ml}$ ont été analysés en double par la technique DEFT et la méthode de référence (norme FIL 100). Le coefficient de corrélation entre ces deux méthodes est de 0,914 et l'écarttype résiduel de la régression référence sur DEFT de $0,245 \mathrm{UFC} / \mathrm{ml}$ en $\log _{10}$. $\mathrm{Le}$ coefficient de variation de répétabilité de la technique DEFT est en moyenne de $14,8 \%$, quel que soit le niveau de contamination du lait.

L'analyse de 44 échantillons de lait cru, soit après une agitation standard, soit après une agitation par turbine, a montré que la désagrégation des amas microbiens améliore nettement la précision d'estimation de la technique DEFT. Par ailleurs, on a mis en évidence une influence plus grande de l'opérateur préparant les lames que du lecteur sur les résultats de la technique DEFT; les raisons possibles et les moyens d'y remédier sont également discutés.

Cent quarante-six lames ont été comptées à la fois à l'œil nu et par l'analyseur d'image 40-10 (Foss-Electric). Le coefficient de corrélation obtenu entre les deux comptages est de 0,984 et l'écart-type résiduel de la régression comptage visuel sur comptage par analyseur d'image de 0,109 bactérie/ml en $\log _{10}$. Les résultats du comptage par analyseur d'images sont en moyenne supérieurs à ceux du comptage visuel, cette différence diminuant avec le niveau de contamination des échantillons. Pour chaque classe de niveau de contamination du lait, nous avons calculé le nombre minimum de champs qui doivent être examinés par l'analyseur d'images pour obtenir une précision de 1,5 à $2 \%$ en valeur logarithmique.

Mots clés : Lait cru - Dénombrement microbien - DEFT - Microscopie épifluorescente - Comptage semi-automatique - Analyseur d'images - Précision. 


\section{Summary}

Assessment of the Direct Epifluorescent Filter Technique (DEFT) with a visual or an image analyser counting for the enumeration of the total flora in raw milk

The Direct Epifluorescent Filter Technique (DEFT) is a rapid method which associates the membrane filtration technique and the epifluorescent microscopy for counting micro-organisms in milk.

A series of 167 raw milk samples, ranging from $7.10^{3}$ to $4.10^{6} \mathrm{CFU} / \mathrm{ml}$ was analyzed in duplicate by the DEFT method and the reference standard plate count method (SPC). The coefficient of correlation between DEFT and SPC is 0.914 and the accuracy expressed by the residual standard deviation from the regression SPC on DEFT, is $0.245 \mathrm{CFU} / \mathrm{ml}$ in $\log _{10}$. The coefficient of variation of repeatability averages $14.8 \%$ and is nearly constant at the various milk levels of contamination.

The analysis of 44 milk samples submitted either to the "standard" shaking or blended with an Ultra-Turrax during 30 sec. at 20000 r.p.m. showed that the disruption of microbial clumps improved the accuracy of the DEFT. Moreover, the DEFT counts are affected more by the operator preparing the slides than by the reader; possible reasons and ways to overcome these discrepancies are discussed.

One hundred forty six slides were examined both visually and by the Image Analyzer 40-10 (Foss Electric). The coefficient of correlation between the two counting procedures is 0.984 and the residual standard deviation from the regression of the visual count over the semi-automated count is 0.109 bacteria/ $\mathrm{ml}$ in $\log _{10}$. The average semi-automated DEFT count is higher than the visual DEFT count but this difference becomes proportionnaly less as the count increases. According to the level of contamination of the milk samples, we have calculated the minimum number of fields which have to be examined to fit an accuracy of 1.5 to $2 \%$.

Key words : Raw milk - Microbial count - DEFT - Epifluorescent microscopy - Semiautomated counting - Image analyzer - Accuracy.

\section{Introduction}

Les méthodes normalisées de numération de la flore totale du lait cru sont basées sur le dénombrement des micro-organismes capables de former des colonies dans un milieu gélosé nutritif non sélectif après une incubation de $72 \mathrm{~h}$ à $30^{\circ} \mathrm{C}$ en aérobiose. Ce temps de réponse très long représente un inconvénient pour l'industriel qui désire connaître rapidement la qualité bactériologique de la matière première mise en cuvre.

Pour répondre à ce besoin de rapidité de réponse, diverses techniques rapides d'estimation de la qualité bactériologique du lait sont proposées actuellement et ont fait l'objet de revues (Pettipher, 1981 ; Bossuyt, 1982 ; O'Toole, 1983 ; O'ConNor, 1984). Parmi ces méthodes, la technique DEFT (Direct Epifluorescent Filter Technique), dont le temps de réponse est de 20 à $30 \mathrm{~min}$, est une méthode de numération directe par microscopie en épifluorescence développée récemment par PetTipher et al. (1980). Cette technique est une amélioration de la technique 
de Breed et Brew (1916) qui porte sur deux points essentiels : une concentration des bactéries par filtration sur une membrane, de façon à augmenter le seuil de détection de la technique, et une coloration des micro-organismes par un fluorochrome pour faciliter le comptage au microscope et son automatisation.

L'utilisation de la technique DEFT pour la numération de la flore totale du lait cru a déjà fait l'objet de travaux (PETTIPHer et al., 1980 et 1983 ; BECK et HeHIR, 1982 ; SuHren et HEESCHEN, 1984). Ces auteurs ont généralement établi la répétabilité de la méthode ainsi que sa corrélation avec la méthode de référence, avec des coefficients de corrélation supérieurs à 0,90 ; mais aucune de ces études ne précise l'ensemble des paramètres de la justesse (précision d'estimation et exactitude de calibrage). Le premier objectif de notre étude a donc été d'établir expérimentalement toutes les caractéristiques analytiques de la DEFT (répétabilité et justesse), selon le schéma proposé par GrapPIN (1976).

Ensuite, compte tenu du principe de la DEFT, nous avons étudié l'incidence de deux facteurs susceptibles de modifier ses caractéristiques : 1'origine des échantillons (laits de troupeaux et laits de citernes de ramassage) et le degré de dispersion des amas microbiens. Comme les études précédentes insistent généralement sur le rôle du comptage des lames sur la répétabilité de la DEFT, nous avons voulu déterminer quelle était également l'importance de l'opérateur effectuant la préparation des lames.

Enfin, en raison de la fatigue de l'opérateur au microscope, cette technique ne permet pas d'atteindre une cadence supérieure à 30-40 échantillons/jour/opérateur. PetTipher et Rodrigues (1982 b) ont alors déjà montré l'intérêt d'une automatisation du comptage des lames; nous nous attacherons dans une troisième partie à établir les caractéristiques analytiques d'un comptage par analyseur d'images.

\section{Matériel et méthodes}

\section{A. Origine des échantillons}

Cent soixante-sept échantillons de lait cru ont été prélevés et conservés à $0{ }^{\circ} \mathrm{C}$, au plus 24 heures après le prélèvement, jusqu'au moment de l'analyse. Parmi ces échantillons, 82 provenaient du mélange du lait d'une seule traite, 20 du mélange habituel du lait de quatre traites et 65 des citernes de ramassage d'une entreprise laitière.

\section{B. Mode d'agitation des échantillons}

Chaque échantillon était soumis, avant analyse, à une agitation standard consistant en 25 mouvements aller-retour d'une amplitude de $25-30 \mathrm{~cm}$ effectués en 7 à 10 secondes (ANON., 1972).

Par ailleurs, pour étudier l'incidence du mode d'agitation des échantillons sur les caractéristiques analytiques de la technique DEFT, 44 échantillons ont été répartis, après homogénéisation, en deux fractions de $25 \mathrm{ml}$ dans des flacons de 
$60 \mathrm{ml}$ : l'une d'elles subissait une agitation standard et l'autre fraction une agitation pendant 30 secondes à l'aide d'un homogénéisateur à turbine (Ultra-Turrax, type TP $18 / 10$ ) tournant à 20000 tours/minute. Ce dernier mode d'agitation est actuellement celui qui assure la meilleure dispersion des amas microbiens (RICHARD, 1980 ; RICHARD et al., 1981).

\section{La technique DEFT}

Cette technique a déjà été décrite par PetTipher et al. (1980) ; nous rappellerons ici toutefois les principales étapes.

\section{Préparation des lames}

Avant analyse, tous les réactifs sont filtrés pour assurer leur stérilisation et l'élimination des particules en suspension.

Le mélange, constitué par $2 \mathrm{ml}$ de lait, $0,5 \mathrm{ml}$ d'enzyme trypsine (Bactotrypsine, Difco) et $2 \mathrm{ml}$ de Triton X 100 à la concentration de $0,5 \%$, est mis à incuber à $50^{\circ} \mathrm{C}$ pendant 10 minutes dans un tube à vis, de façon à détruire les cellules somatiques et à permettre le passage des globules gras à travers la membrane en polycarbonate.

Une membrane en polycarbonate (Nuclepore) de $0,6 \mu \mathrm{m}$ de porosité et $25 \mathrm{~mm}$ de diamètre est montée, côté brillant vers le haut, sur le système de filtration relié à une pompe à vide. N'ayant pas de régulateur de vide, la dépression obtenue variait entre 50 et $80 \mathrm{kPa}$. Le filtre est d'abord préchauffé pour faciliter la filtration, par addition de $5 \mathrm{ml}$ de Triton X 100 à la concentration de $0,1 \%$ à $50^{\circ} \mathrm{C}$. Après filtration du lait, le rinçage du tube à vis et du filtre est réalisé grâce à 5 ml de Triton X 100 à la concentration de $0,1 \%$.

La coloration des bactéries est ensuite effectuée par addition de $2,5 \mathrm{ml}$ d'une solution d'acridine orange (Laboratoires Raymond) à la concentration de 0,025\% dans un tampon citrate $\mathrm{pH}$ 6,6. Ce fluorochrome a la propriété de colorer spécifiquement en vert l'acide désoxyribonucléique (ADN) et en orange l'acide ribonucléique (ARN) des cellules (Von BERTALANFFY et BICKIS, 1956); il en résulte théoriquement que seules les bactéries « actives», riches en ARN, prennent une coloration orange (НоввIE et al., 1977). Après un temps de contact de 4 minutes, le colorant est éliminé et la membrane est rincée avec $2,5 \mathrm{ml}$ de tampon citrate $\mathrm{pH} 3$ puis $2,5 \mathrm{ml}$ d'isopropanol. Ces deux rinçages sont effectués rapidement de façon à éviter une décoloration des bactéries. La membrane est ensuite séchée à l'air et disposée dans une immersion d'huile non fluorescente entre lame et lamelle.

\section{Observation miscroscopique}

Les lames ont été examinées le jour de leur préparation à l'aide d'un microscope Olympus BHS équipé d'un dispositif d'épifluorescence, d'un objectif S Plan achromatique $100 \mathrm{X}$ à immersion et d'un oculaire grand champ WHX10X équipé d'un quadrillage. Le facteur de multiplication de ce microscope, permettant de calculer le nombre de bactéries par millilitre de lait, est, dans les conditions expérimentales, de 5325 .

Les amas de cellules colorées en orange ont été comptés dans des champs microscopiques pris au hasard, selon les normes définies par PetTipher et al. (1980). 


\begin{tabular}{c|c}
\hline Nombre de champs à compter & Nombre moyen d'amas par champ \\
\hline 15 & $0-10$ \\
10 & $11-25$ \\
6 & $26-50$ \\
3 & $51-75$ \\
2 & $76-100$ \\
\hline
\end{tabular}

Pour les lames ayant plus de 100 amas par champ, nous n'avons pas effectué de dilution, comme le recommandent ces auteurs ; nous avons examiné seulement $30 \%$ de la surface totale du champ, le nombre de champs étant fixé arbitrairement à 6 . Un amas est défini comme une cellule ou un groupe de cellules séparés des autres par une distance égale ou supérieure à deux fois le plus petit diamètre des deux cellules les plus proches l'une de l'autre (ANON., 1972).

\section{Comptage semi-automatique des lames à l'aide d'un analyseur d'images}

Nous avons utilisé l'analyseur d'images 40-10 commercialisé par la Société Foss-Electric France. Le système comprend une caméra qui transmet l'image observée dans le microscope à l'analyseur d'images et à un écran vidéo qui permet à l'opérateur d'observer l'image transmise par la caméra. L'analyseur d'images compte les points lumineux par comparaison à une échelle de niveaux de gris ; il est contrôlé par un microprocesseur qui traite les données fournies. Dans ces conditions expérimentales, le facteur de multiplication du microscope était de 21780 ; la surface de champ utile correspondait à $25 \%$ du champ total du microscope.

Comme Pettipher et Rodrigues (1982 b), nous avons réalisé, pour chaque lame observée par l'analyseur d'images, le comptage de vingt champs choisis au hasard, quel que soit le niveau de contamination du lait.

\section{E. Numérations microbiennes}

Les échantillons de lait cru ont été soumis au dénombrement des bactéries aérobies mésophiles selon la norme provisoire FIL 100 (ANON., 1981) ; les résultats sont exprimés en unités formant colonies par millilitre de lait (UFC/ml).

\section{F. Protocole expérimental et calculs statistiques}

\section{Paramètres analytiques}

Les caractéristiques analytiques de la technique DEFT et du comptage par analyseur d'images ont été déterminées à partir des analyses en double des échantillons par chacune des techniques. La répétabilité et la justesse ont été calculées 
selon les modèles décrits précédemment pour les méthodes indirectes d'analyse du lait (GRAPPIN, 1976).

Influence de l'opérateur, de la lame et du lecteur sur les résultats de la technique DEFT

Trente-quatre échantillons de lait (19 agités de façon standard et 13 par turbine) ont été analysés par la technique DEFT par deux opérateurs selon le protocole expérimental de la figure 1 . Sur chacun des échantillons, chaque opérateur a réalisé deux lames; en pratique, l'un des opérateurs maintenait une dépression plus forte lors de la filtration. Chaque lame a été lue à deux reprises par chacun des deux opérateurs, selon les règles définies plus haut.

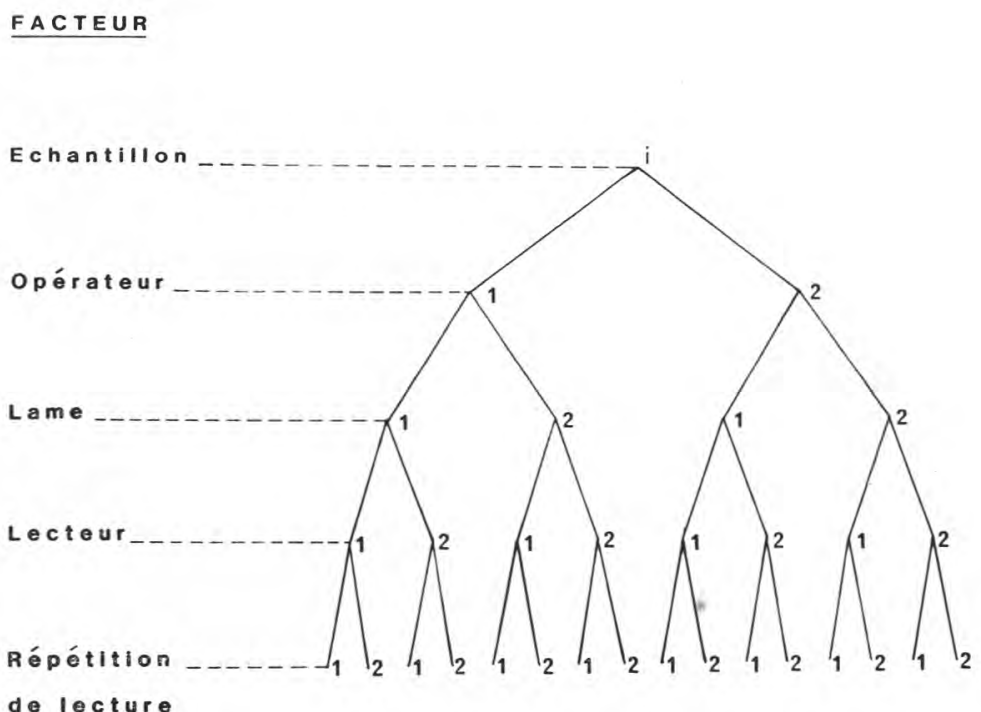

Fig. 1

Plan de l'expérimentation sur l'influence de l'opérateur, de la lame et du lecteur sur les résultats de la technique DEFT.

Plan of the experiment on the influence of the operator, the slide and the reader on the DEFT counts.

Pour chaque classe d'échantillons (agitation standard et agitation turbine), les résultats de dénombrement (16 résultats/échantillon) ont été soumis à une analyse de variance à modèle croisé hiérarchisé puisqu'il n'existe aucune correspondance entre les lames réalisées par les deux opérateurs sur les différents échantillons. Les facteurs pris en compte dans le modèle sont les suivants : facteurs échantillon, opérateur, lame, lecteur et interaction échantillon $\times$ opérateur. 


\section{Résultats et discussion}

A. Caractéristiques analytiques de la technique DEFT

\section{Répétabilité}

Pour éviter une transformation logarithmique des données, la répétabilité des mesures a été calculée pour des classes de niveaux de contamination du lait à l'intérieur desquelles les variances de répétabilité sont supposées homogènes. Le tableau I montre les différents coefficients de variation de répétabilité $\left(\mathrm{CV}_{\mathbf{r}}\right.$ calculés pour la méthode de référence et la technique DEFT. En dehors de la classe des laits contenant moins de 50000 micro-organismes $/ \mathrm{ml}$, on observe un $\mathrm{CV}_{\mathrm{r}}$ relativement constant, quel que soit le niveau de contamination du lait.

TABLEAU I

Répétabilité de la technique DEFT et de la méthode de référence en fonction du niveau de contamination des échantillons de lait

Repeatability of the DEFT and the reference standard plate count method for various milk samples levels of contamination

\begin{tabular}{|c|c|c|c|c|c|c|}
\hline \multirow{2}{*}{$\begin{array}{c}\text { Nombre de } \\
\text { micro-organismes } / \mathrm{ml}\end{array}$} & \multicolumn{3}{|c|}{ Référence } & \multicolumn{3}{|c|}{ DEFT } \\
\hline & $n^{(a)}$ & moyenne & $\mathrm{CV}_{r}{ }^{(\mathrm{b})}$ & $n^{(a)}$ & moyenne & $C v_{r}{ }^{(b)}$ \\
\hline moins de $5.10^{4}$ & 25 & 26000 & 19,0 & 33 & 28000 & 19,8 \\
\hline $5.10^{4}-10^{5}$ & 30 & 72000 & 11,5 & 30 & 75000 & 14,1 \\
\hline $10^{5}-2.10^{5}$ & 38 & 150000 & 7,2 & 34 & 150000 & 12,1 \\
\hline $2.10^{5}-5.10^{5}$ & 27 & 320000 & 10,1 & 23 & 320000 & 18,2 \\
\hline $5.10^{5}-10^{6}$ & 27 & 690000 & 8,9 & 30 & 670000 & 11,3 \\
\hline plus de $10^{6}$ & 202 & 2000000 & 6,8 & 171 & 900000 & 13,1 \\
\hline
\end{tabular}

(a) n: nombre d'échantillons

(b) $\mathrm{CV}_{\mathrm{r}}$ : coefficient de variation de répétabilité.

Pour l'ensemble des échantillons, le $\mathrm{CV}_{\mathrm{r}}$ moyen est de $10,5 \%$ pour la méthode de référence et de $14,8 \%$ pour la technique DEFT. La répétabilité trouvée pour cette technique est donc très proche de la valeur observée pour la méthode Thompson (Grappin et Jeunet, 1974). Par contre, elle est nettement meilleure que celle donnée pour cette même technique dans les études précédentes puisque Pettipher et al. (1983) et ensuite Suhren et Heeschen (1984) ont observé des $\mathrm{CV}_{\mathrm{r}}$ de 19 à $20 \%$. 


\section{Justesse}

La comparaison entre les résultats de la technique DEFT et de la méthode de référence a été établie à partir des logarithmes décimaux des moyennes des deux déterminations faites sur chaque échantillon pour chacune des méthodes (fig. 2). L'équation de régression linéaire calculée sur 167 échantillons contenant entre $7.10^{3}$ et $4.10^{6} \mathrm{UFC} / \mathrm{ml}$ est la suivante :

$\log _{10}$ (Réf.) $=0,9255 \times \log _{10}$ (DEFT) $+0,4362$ avec un écart-type résiduel $\left(s_{y, x}\right)$ de 0,245 et un coefficient de corrélation linéaire de 0,914. De ces résultats, on peut déduire les deux paramètres essentiels de la justesse : la précision d'estimation et l'exactitude de calibrage de la technique.

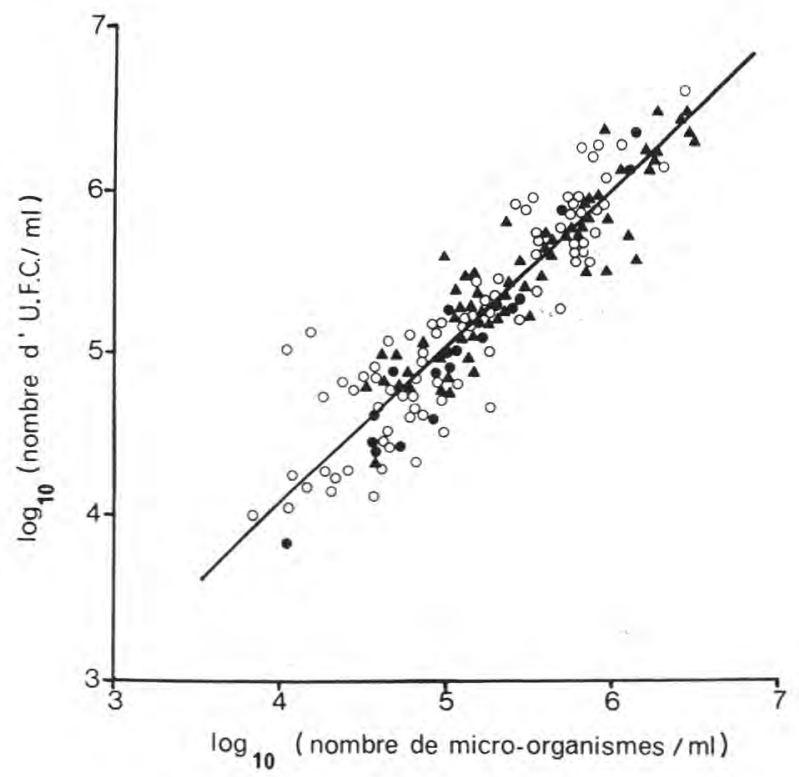

Fig. 2

Relation entre les résultats de dénombrement de la flore totale du lait cru par la technique $D E F T$ (en abscisse) et par la méthode de référence (en ordonnée) : (0) mélange d'une traite, $(\bullet)$ mélange du lait de quatre traites, $(\mathbf{\Delta})$ laits de citerne, - : droite de régression. Relationship between DEFT counts (abscissa) and reference standard plate counts (ordinate) of raw milk samples: (O) farm milks (mixture of one milking), ( $\bullet$ ) farm milks (mixture of four milkings), ( $\mathbf{\Delta})$ bulked tanker milks, - : fitted regression line.

\section{Précision d'estimation}

Définie comme l'étroitesse de l'accord entre la méthode de référence et la technique DEFT après élimination éventuelle d'un défaut de calibrage, la précision d'estimation est donnée par les limites de l'intervalle de confiance à l'intérieur duquel se situe, avec une probabilité de $95 \%$, la vraie valeur donnée par la méthode de référence.

Cet intervalle est égal à $\pm \mathrm{t} \times s_{v, x}$ soit dans le cas présent : $1,96 \times 0,245=$ $\pm 0,479$. A partir de ce résultat, le tableau II indique, directement en nombre d'UFC/ml, ia précision d'estimation de la technique DEFT pour trois niveaux 
TABLEAU II

Précision d'estimation de la technique DEFT à différents niveaux de contamination microbienne du lait

Accuracy of the DEFT at various milk sample levels of contamination

\begin{tabular}{|c|c|}
\hline $\begin{array}{c}\text { Résultats DEFT } \\
\left(\text { nombre de bactéries } / \mathrm{ml} \times 10^{3}\right)\end{array}$ & $\begin{array}{c}\text { Lirrites de confiance * } \\
\left(\mathrm{UFC} / \mathrm{ml} \times 10^{3}\right)\end{array}$ \\
\hline 50 & $18-139$ \\
100 & $36-277$ \\
500 & $180-1390$ \\
\hline
\end{tabular}

* Limites de confiance $(P=0,95)$ de la valeur estimée du nombre d't $\mathrm{FC} / \mathrm{ml}$

de qualité bactériologique du lait. En raison de la transformation logarithmique initiale, les limites des intervalles sont dissymétriques par rapport à la moyenne.

\section{Exactitude de calibrage}

Pour que la méthode soit exactement calibrée, il faut que le coefficient de régression $\mathrm{b}$ de l'équation de régression $\log _{10}$ (Réf) $=\mathrm{b} \times \log _{10}(\mathrm{DEFT})+\mathrm{a}$ ne soit pas statistiquement différent de 1 et que l'ordonnée à l'origine a ne soit pas différente de zéro, cette dernière condition pouvant être remplacée par l'égalité des moyennes des deux variables.

On observe sur la figure 2 que deux échantillons s'écartent très nettement du nuage de points. Nous avons donc choisi de les éliminer pour étudier le calibrage de la droite. Dans ce cas, l'équation de régression devient :

$$
\log _{10}(\text { Réf })=0,9615 \times \log _{10}(\text { DEFT })+0,2367
$$

Avec une erreur standard $s_{\mathrm{b}}=0,0302$, le cofficient de régression $\mathrm{b}=$ $0,9615 \pm 0,0592$ n'est pas statistiquement différent de 1,000 ; par ailleurs, les deux moyennes $\log _{10}$ (Réf) $=5,284$ et $\log _{10}($ DEFT $)=5,238$ ne sont pas statistiquement différentes. Par conséquent, la technique DEFT est parfaitement calibrée et les résultats peuvent être utilisés directement sans avoir recours à un facteur de correction.

\section{B. Facteurs influençant la relation entre la technique DEFT et la méthode de référence}

\section{Origine des échantillons}

Le tableau III présente les caractéristiques de la technique DEFT, calculées sur les 102 échantillons de laits de producteurs d'une part et sur les 62 échantillons de laits de citernes de ramassage d'autre part. On observe tout d'abord que les ordonnées à l'origine et les coefficients de régression des droites obtenues pour les deux classes d'échantillons ne sont pas statistiquement différents à 


\section{TABLEAU III}

Influence de l'origine des laits sur la relation entre les résultats de la technique $\operatorname{DEFT}(x)$ et de la méthode de référence $(y)$

Influence of the origin of the milk samples on the relationship between the DEFT $(x)$ and the standard plate counts $(y)$

\begin{tabular}{|c|c|c|c|c|c|}
\hline Origine des laits & $n^{(a)}$ & move & b) $\quad r(c)$ & $\begin{array}{l}\text { Equation de la } \\
\text { droite de regression }\end{array}$ & $\begin{array}{l}\text { Ecart-type } \\
\text { résiduel }\end{array}$ \\
\hline Producteurs. & 102 & 5,14 & 0,902 & $y=0,949+0,323 x$ & 0,267 \\
\hline Citernes & 65 & 5,50 & 0,917 & $y=0,857+0,823 x$ & 0,202 \\
\hline
\end{tabular}

(a) it: nombre d'cchanitilons

(b) moyenne des resultats de la méthode de réference

(c) $r$ : coefficient de corrélation

$\mathbf{P}=0,95$. Cependant, malgré des coefficients de corrélation sensiblement identiques, la précision d'estimation de la technique DEFT est nettement meilleure dans le cas des laits de citerne que dans celui des laits de producteurs.

Quelques hypothèses peuvent être émises pour expliquer cet écart. En effet, les laits de citerne ont déjà fait l'objet d'une conservation prolongée à basse température, entraînant la prolifération des seuls Pseudomonas psychrotrophes (RICHARD, 1981). La bonne relation entre les deux méthodes peut alors résulter de deux facteurs : une flore relativement homogène et des cellules bactériennes fortement colorées en orange puisqu'elles sont situées en phase exponentielle de croissance (Pettipher et al., 1980). Par contre, les échantillons de laits de producteurs provenaient, pour la majorité d'entre eux, du mélange du lait d'une seule traite qui n'avait donc fait l'objet d'aucune conservation. Ainsi, il est vraisemblable que des cellules bactériennes, bien que capables de pousser sur un milieu nutritif, soient cependant colorées en vert en raison de leur mauvais état physiologique sur la peau des mamelles ou dans le matériel de traite. A l'inverse, des cellules non viables à la suite, par exemple, du traitement thermique occasionné par le nettoyage du matériel de traite, peuvent prendre une coloration orange (Pettipher et Rodrigues, 1981 et 1982 a ; Hunter et Mac Corquodale, 1983). De plus, la conservation des échantillons pendant $24 \mathrm{~h}$ à $0^{\circ} \mathrm{C}$ a pu provoquer des lésions de certaines cellules bactériennes mésophiles strictes et ainsi modifier leurs propriétés de coloration par le fluorochrome.

\section{Mode d'agitation des échantillons}

Le tableau IV présente la répétabilité de la méthode de référence et de la technique DEFT, pour chaque mode d'agitation des échantillons. Contrairement à ce qu'avaient noté RICHARD et al. (1981) pour la méthode Thompson, on observe une répétabilité un peu moins satisfaisante de la méthode de référence, après une agitation vigoureuse des échantillons. Par contre, Ie $\mathrm{CV}_{\mathrm{r}}$ de la technique DEFT est nettement amélioré avec l'agitation par turbine. Cela peut aisément être attribué à une désagrégation. importante des amas microbiens par la turbine 


\section{TABLEAU IV}

Influence du mode d'agitation des échantillons de lait cru sur la répétabilité de la technique DEFT et de la méthode de référence

\section{Influence of the sample shaking method on the repeatability} of the DEFT and the standard plate count method

\begin{tabular}{|l|l|l|l|l|l|}
\hline \multirow{2}{*}{ Mode d'agitation } & \multirow{2}{*}{$n^{(a)}$} & \multicolumn{2}{|c|}{ Méthode de référence } & \multicolumn{2}{|c|}{ Technique DEFT } \\
\cline { 3 - 6 } & & Moyenne & $\mathrm{CV}_{r}{ }^{(\mathrm{b})}$ & Moyenne & $\mathrm{CV}_{r}^{(\mathrm{b})}$ \\
\hline Standard & 44 & 210000 & 11,0 & 190000 & 13,0 \\
Turbine & 44 & 460000 & 13,1 & 340000 & 9,6 \\
\hline
\end{tabular}

(a) $n$ : nombre d'échantillons.

(b) $\mathrm{CV}_{\mathrm{r}}$ : coefficient de variation de répétabilité.

(Richard, 1980 ; Piton et Richard, 1983). Ainsi, après ce mode d'agitation, plus de $95 \%$ des amas de bactéries à Gram négatif sont sous forme d'amas d'une à deux cellules; le comptage des amas au microscope est donc beaucoup plus aisé.

Ces raisons permettent également d'expliquer l'amélioration de la relation entre les deux méthodes avec une agitation vigoureuse (fig. 3). Ainsi, la précision d'estimation de la méthode DEFT passe, en valeur logarithmique, de $\pm 0,664$ pour l'agitation standard à $\pm 0,392$ pour l'agitation par turbine.

3. Importance respective des facteurs opérateur, lame et lecteur sur les résultats de la technique DEFT

Le tableau $\mathrm{V}$ présente les analyses de variance des résultats de dénombrement par la technique DEFT, pour les 19 et 13 échantillons agités de façon standard ou par turbine respectivement.

Les niveaux de contamination des laits analysés varient entre $5.10^{3}$ et $3.10^{6} \mathrm{UFC} / \mathrm{ml}$. Pour les deux classes d'échantillons, les facteurs opérateur et lame influencent de façon significative les résultats de dénombrement, avec une interaction échantillon $x$ opérateur. Cela signifie qu'il existe des différences entre manipulateurs mais que celles-ci varient en fonction de l'échantillon; il existe également une différence entre les deux lames réalisées par le même manipulateur sur le même échantillon. A l'inverse, l'effet lecteur n'est pas significatif. La variance résiduelle peut être assimilée à une variance de reproductibilité, bien que les analyses aient été réalisées dans le même laboratoire et sur le même microscope. Comme cela l'a déjà été observé pour le coefficient de variation de répétabilité (tabl. 4), cette variance est nettement plus faible dans le cas des laits agités vigoureusement; en outre, elle est inférieure à la valeur limite admise de 0,0050 pour la variance de répétabilité de la méthode classique de dénombrement (ANON., 1972). 


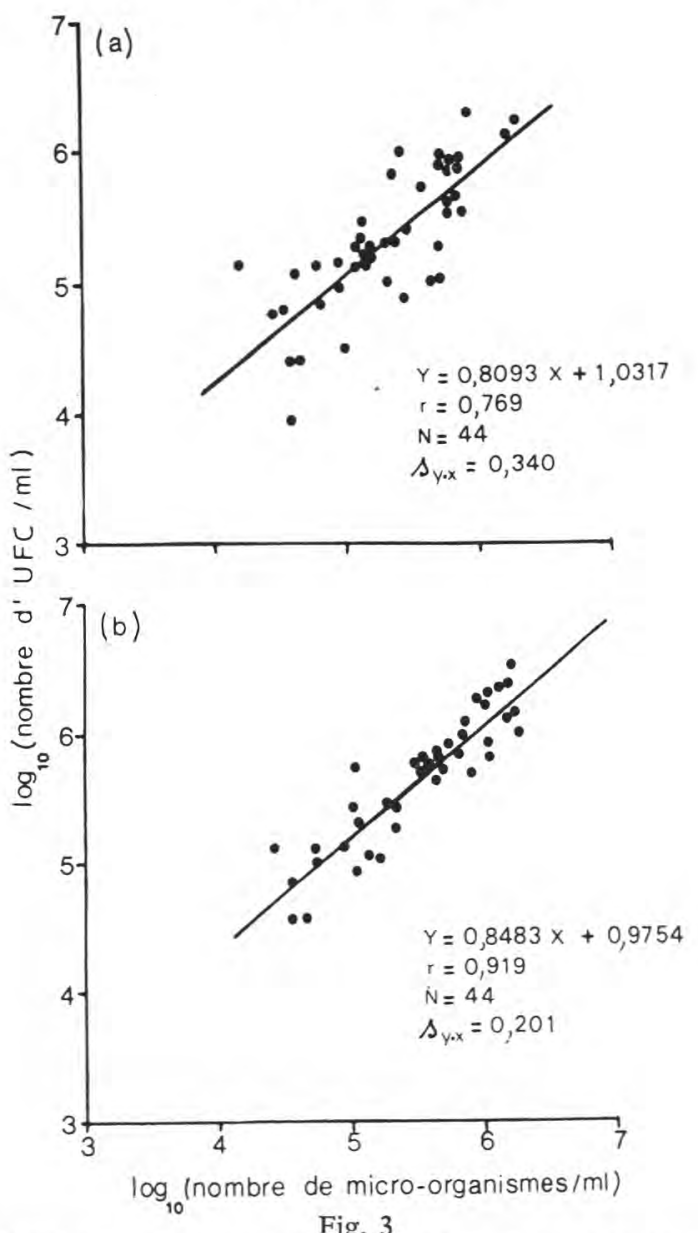

Influence du mode d'agitation des échantillons de lait cru sur la relation entre les résultats de la techniqu DEFT (en abscisse) et de la méthode de référence (en ordonnée).
(a) agitation standard.
(b) agitation par turbine.

Influence of the sample shaking method on the relationship between the DEFT counts (abscissa) and the reference standard plate counts (ordinate).
(a) standard shaking.
(b) shaking with a mixer.

Notre étude semble donc entrer en contradiction avec les travaux antérieurs qui insistent généralement sur l'importance des erreurs de lecture. Les raisons évoquées sont diverses : une mauvaise répartition des bactéries sur la membrane (Jones et Simon, 1975); une augmentation considérable de la variabilité du nombre de bactéries par champ lorsque ce nombre diminue (KIRCHMAN et al., 1982) ; des microscopes et des systèmes d'éclairage différents entre laboratoires (Pettipher et al., 1983) ; une répétabilité entre lecteurs établie sur une forte proportion d'échantillons à faible niveau de contamination (SUHREN et HeEschen, 1984). Le rôle peu important attribué à l'effet lecture au cours de nos essais peut ainsi être expliqué par deux points : un comptage réalisé le même jour sur le même microscope et une même technique de comptage pour les deux lecteurs. D'autre part, nous avons mis en évidence l'importance de la préparation des lames 


\section{TABLEAU V}

Analyse de variance des résultats des dénombrements réalisés par la technique DEFT

Analysis of variance of the DEFT counts of bacteria

(a) Echantillons de lait soumis à l'agitation standard Milk samples submitted to the "standard"shaking method

\begin{tabular}{|l|c|c|c|}
\hline Source de variation & $\mathrm{N}^{(\mathrm{a})}$ & carré moyen & $\mathrm{F}^{(\mathrm{b})}$ \\
\hline Echantillon & 18 & 7,1037 & $1504^{* *}$ \\
Opérateur & 1 & 0,0221 & $4,68^{*}$ \\
Echantillon x Opérateur & 18 & 0,0123 & $2,60^{* *}$ \\
Lame & 38 & 0,0121 & $2,57^{* *}$ \\
Lecteur & 1 & 0,0146 & 3,09 \\
Résiduelle & 227 & 0,0047 & \\
\hline
\end{tabular}

(b) Echantillons de lait agités pendant $30 \mathrm{~s}$ à l'Ultra-Turrax Milk samples blended with an Ultra-Turrax for $30 \mathrm{~s}$

\begin{tabular}{|l|c|c|c|}
\hline Source de variation & $N^{(a)}$ & carré moyen & $F^{(\mathrm{b})}$ \\
\hline Echantillon & 12 & 4,7470 & $4687^{* *}$ \\
Orérateur & 1 & 0,0821 & $81,06^{* *}$ \\
Echantillon x Opérateur & 12 & 0,0198 & $19,52^{* *}$ \\
Lame & 26 & 0,0064 & $6,28^{* *}$ \\
Lecteur & 1 & 0,0002 & 0,18 \\
Résiduelle & 155 & 0,0010 & \\
\hline
\end{tabular}

(a) $\mathrm{N}$ : nombre de degré de liberté

(b) F : rapport des variances de Fisher Snedecor

* et ** : significatif à $\mathrm{P}=0,05$ et $\mathrm{P}=0,01$ respectivement.

sur les résultats de la technique DEFT. Quelques hypothèses peuvent être émises comme la coloration plus ou moins intense des micro-organismes ou l'application, lors de la filtration, d'une dépression plus ou moins importante d'un opérateur à l'autre, ou d'un essai à l'autre pour le même opérateur. En effet, nous avons pu constater, sur certains laits, des problèmes de coloration rendant le comptage difficile. Par ailleurs, d'après de nombreux auteurs (Jones et Simon, 1975; HobBie et al., 1977 ; BeCK et HeHIR, 1982), les filtres de porosité $0,6 \mu$ ne permettent pas de retenir, à la surface du filtre, toutes les bactéries de petite taille qui se trouvent alors imbriquées dans la trame du filtre; il est donc vraisemblable qu'une filtration rapide sous un vide poussé entraîne une succion plus importante des bactéries. 


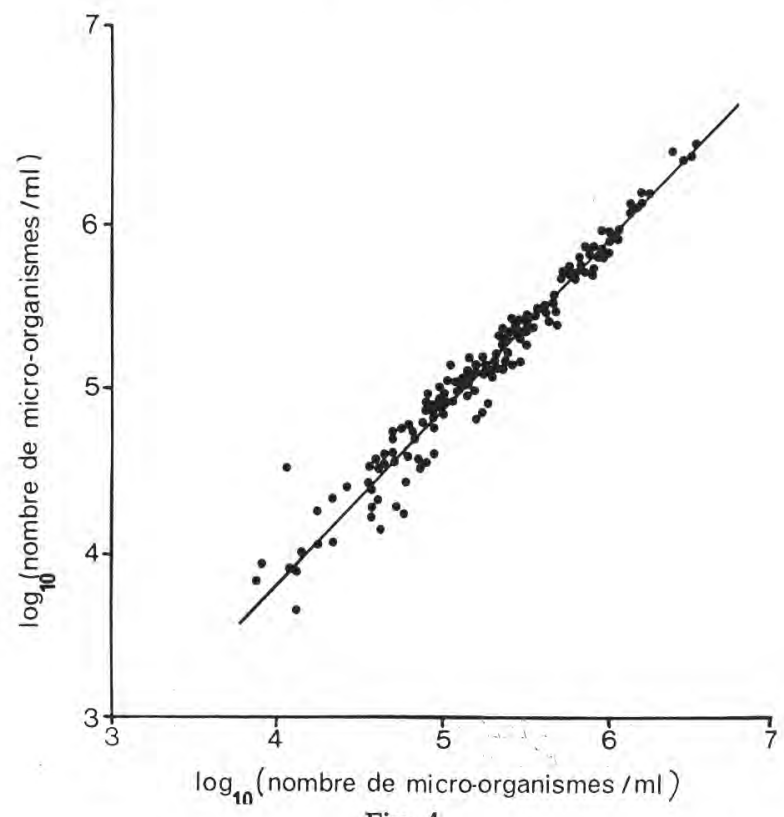

Fig. 4

Relation entre les résultats de dénombrement de la flore totale du lait cru par la technique DEFT en comptage semi-automatique (en abscisse) et en comptage visuel (en ordonnée).

Relationship between semi-automated DEFT counts (abscissa) and visual DEFT counts (ordinate) of bacteria in raw milk.

\section{Etude du comptage des lames par l'analyseur d'images}

1. Justesse

La comparaison des deux types de comptage a été établie grâce à l'observation de 146 lames obtenues à partir de laits agités de façon standard contenant entre $1,7.10^{4}$ et $3.10^{6} \mathrm{UFC} / \mathrm{ml}$ (fig. 3 ).

L'équation de régression linéaire est la suivante :

$\log _{10}($ comptage visuel $)=1,0607 \times \log _{10}$ (analyseur $)-0,4240$

avec un écart-type résiduel de 0,1086 et un coefficient de corrélation de 0,984 .

Calibrage du comptage par l'analyseur d'images

Le coefficient de régression de l'équation $b=1,061 \pm 0,031$ est statistiquement différent de 1 au seuil $\mathrm{P}=0,95$; les deux moyennes $\log _{10}$ (comptage visuel $)=5,132$ et $\log _{10}($ comptage analyseur $)=5,238$ sont également statistiquement différentes à $\mathrm{P}=0,99$. Pour corriger les résultats de l'analyseur d'image du défaut de calibrage, il est nécessaire d'appliquer à ces résultats l'équation de calibrage donnée ci-dessus ; une manière plus simple mais moins rigoureuse consiste à appliquer un facteur de correction (FC) correspondant au rapport des moyennes des résultats des deux comptages. Dans notre cas, il est égal à 1,27 , ce qui signifie que les valeurs obtenues par l'analyseur doivent être divisées par 1,27 pour avoir les résultats en comptage visuel. 


\section{Précision d'estimation}

La précision d'estimation du comptage par l'analyseur d'images est de $\pm 1,96 \times 0,1086= \pm 0,213$. Cependant, on observe, sur le tavleau VI, qu'elle varie de façon très nette avec le niveau de contamination du lait. De ces résultats, on peut aisément conclure que le nombre de champs fixé arbitrairement à 20 est probablement nettement insuffisant dans le cas des laits contenant moins de $10^{5} \mathrm{UFC} / \mathrm{ml}$.

\section{TABLEAU VI}

Relation entre les résultats du comptage semi-automatique ( $x$ ) et du comptage visuel $(y)$ pour différents niveaux de contamination du lait

Relationship between the semi-automated $(x)$ and visual $(y)$ DEFT counts at various milk samples levels of contamination

\begin{tabular}{|l|c|c|c|c|c|}
\hline \multirow{2}{*}{$\begin{array}{l}\text { Nombre de } \\
\text { bactéries/ml }\end{array}$} & \multirow{2}{*}{$N^{(a)}$} & \multicolumn{4}{|c|}{ Paramètres statistiques (b) } \\
\cline { 3 - 6 } & & $r$ & Equation de régression & $s_{\mathrm{y}, \mathrm{x}}$ & $\Delta$ \\
\hline moins de $10^{5}$ & 59 & 0,907 & $y=0,998 x-0,139$ & 0,147 & 0,151 \\
$10^{5}-5.10^{5}$ & 53 & 0,908 & $y=0,800 x+0,976$ & 0,068 & 0,096 \\
$>5.10^{5}$ & 34 & 0,981 & $y=1,049 x-0,340$ & 0,045 & 0,043 \\
TOTAL & 146 & 0,984 & $y=1,061 \times-0,424$ & 0,109 & 0,106 \\
\hline
\end{tabular}

(a) $N$ : nombre de lames examinées

(b) $r$ : coefficient de corrélation

$\Delta_{y, x}:$ écart-type résiduel

$\Delta$ : différence entre les deux moyennes $(\bar{x}-\bar{y})$

Par ailleurs, quel que soit le niveau de contamination du lait, on observe une surestimation du nombre de bactéries par comptage automatique ; cette surestimation est d'autant plus grande que le niveau de contamination du lait baisse. Pettipher et Rodrigues (1982 b) avaient d'ailleurs déjà fait les mêmes observations. Comme ces derniers, nous pouvons attribuer cet écart au comptage de particules (colorant, sédiments).

\section{Détermination du nombre de champs à examiner par l'analyseur d'images}

Nous avons choisi arbitrairement de réaliser le comptage des amas microbiens dans 20 champs pris au hasard, cela dans le but de calculer, pour chaque niveau de contamination du lait, la répétabilité du comptage par l'analyseur d'images ; pour ce calcul, chaque détermination correspond à la moyenne des comptages dans 5 champs microscopiques. Cela nous permettait ensuite de déter- 
miner par calcul le nombre de champs qu'il faudrait examiner pour obtenir une précision d'estimation satisfaisante pour chaque niveau de contamination du lait.

L'intervalle de confiance d'une moyenne est donné par la formule suivante :

$$
\mathrm{m}-\frac{\text { t.s }}{\sqrt{\mathrm{n}}} \leqslant \mu \leqslant \mathrm{m}+\frac{\text { t.s }}{\sqrt{\mathrm{n}}}
$$

où $\mathrm{m}$ est une estimation de la moyenne $\mu$ de $\mathrm{n}$ comptages semi-automatiques réalisés sur une lame, $\mathrm{s}$ est une estimation de l'écart-type de répétabilité de ces comptages et $\mathrm{t}$ la valeur de la distribution de Student à $\mathrm{n}-1$ degrés de liberté (ddl). De cette formule, on peut donc déduire le nombre de champs (n) à examiner pour obtenir la précision souhaitée en $\%(\mathrm{p})$ pour chaque niveau de contamination du lait, soit $\mathrm{n}=\mathrm{t}^{2} \frac{\mathrm{s}^{2} \times 10^{4}}{\mathrm{~m}^{2} \cdot \mathrm{p}^{2}}$;

en effet la différence maximale $\mathrm{m}-\mu(\grave{\mathrm{a}} \mathrm{P}=0,95)$ est égale à $\mathrm{p} \times \mathrm{m}$. Pour nos calculs, nous avons choisi d'estimer $\mathrm{m}$ à partir de la moyenne de tous les dénombrements réalisés par l'analyseur d'images. A partir de ces données, il suffit de fixer $\mathrm{n}$, de calculer sa valeur théorique à partir de la valeur de la distribution de Student à $\mathrm{n}-1$ ddl et de retenir la valeur la plus proche de la valeur théorique. Le nombre de comptages à réaliser (n) devra donc être multiplié par 5 pour obtenir son équivalence en nombre de champs microscopiques.

Outre les valeurs utilisées pour le calcul, le tableau 7 présente, pour chaque classe de niveau de contamination du lait, le nombre de comptages à réaliser ainsi

\section{TABLEAU VII}

Détermination du nombre de champs microscopiques à examiner par l'analyseur d'images

Determination of the number of miscroscopic fields which have to be examined by the image analyser

\begin{tabular}{|l|l|c|c|c|c|}
\hline $\begin{array}{l}\text { Niveau de } \\
\text { contamination } \\
\text { (en UFC/ml) }\end{array}$ & $\begin{array}{l}\text { Précision } \mathrm{P}^{*} \\
\text { souhaitée } \\
\text { (en \%) }\end{array}$ & $\begin{array}{c}\text { Moyenne } \\
\mathrm{m}\end{array}$ & $\begin{array}{c}\text { Ecart- } \\
\text { type } \\
\mathrm{s}\end{array}$ & $\mathrm{n}^{* *}$ & $\begin{array}{l}\text { Equivalence } \\
\text { en nombre } \\
\text { de champs }\end{array}$ \\
\hline moins de $10^{5}$ & 2 & 4,674 & 0,116 & 8 & 40 \\
$10^{5}-5.10^{5}$ & 1,5 & 5,370 & 0,060 & 5 & 25 \\
plus de $5.10^{5}$ & 1,5 & 6,020 & 0,056 & 4 & 20 \\
\hline
\end{tabular}

* précision souhaitée en valeur logarithmique

* Nombre de comptages à réaliser, calculé à partir de la formule $n=t^{2} \cdot \frac{s^{2} \cdot 10^{4}}{\mathrm{p}^{2} \cdot \mathrm{m}^{2}}$

chaque comptage correspondant à la moyenne de 5 champs. t est la valeur du

I de Student à $n$ - $\mathbf{I}$ degrés de liberté. 
que le nombre correspondant de champs microscopiques. Comme on pouvait s'y attendre, ce nombre augmente lorsque le niveau de contamination diminue. Le nombre de 20 champs choisi était donc satisfaisant pour les échantillons fortement contaminés ; il était par contre nettement insuffisant pour les laits contenant moins de $10^{5} \mathrm{UFC} / \mathrm{ml}$. Ce calcul permet ainsi d'expliquer les observations faites au tableau 6.

\section{Conclusion}

A l'inverse des techniques indirectes physico-chimiques ou immunologiques, les techniques rapides de microscopie en épifluorescence (DEFT, Bacto-Scan) présentent l'avantage de posséder un principe de mesure assez proche de celui des méthodes classiques de numération de la flore totale; il en résulte une limitation de l'incertitude sur l'estimation des valeurs réelles données par une méthode de référence choisie par convention.

Confirmant les travaux antérieurs (Pettipher et al., 1980 et 1983 ; BeCK et Hehir, 1982 ; SuHren et HeEschen, 1984), notre étude montre qu'il existe une relation satisfaisante entre les résultats de la DEFT et de la méthode de référence. Si les écarts sont parfois importants pour les laits de producteurs avec un écart-type résiduel $\mathrm{S}_{y, x}$ de 0,267 , la corrélation s'améliore nettement si l'on analyse des laits de grand mélange $\left(S_{y, x}=0,202\right)$ ou si l'on fait subir aux échantillons une agitation vigoureuse qui provoque une dispersion des amas microbiens et facilite le comptage $\left(S_{y, x}=0,200\right)$. En outre, la précision d'estimation trouvée pour les laits de troupeau est sensiblement identique à celle observée pour le Bacto-Scan sur le même type de laits (GRAPPIN et al., 1985) ; par contre, la répétabilité est nettement moins satisfaisante que dans le cas de cet appareil entièrement automatique.

Pettipher et al. (1983) avaient déjà attribué un rôle important au type de matériel utilisé (microscope et dispositif d'éclairage) sur la reproductibilité de la DEFT. Quant à notre étude, elle souligne l'influence de la préparation des lames sur les résultats de cette technique. Il est vraisemblable qu'une standardisation des opérations réalisées lors de cette préparation (rapidité de filtration en particulier) aurait permis d'améliorer sa répétabilité et sa précision.

D'autre part, parmi les avantages de la technique DEFT, celui d'une possibilité d'automatisation des comptages est un atout important, permettant d'augmenter la cadence des analyses. Comme l'avaient déjà fait PetTIPHER et RoDrigues (1982 b), nous avons établi qu'avec un choix judicieux du nombre de champs microscopiques, un comptage des micro-organismes par un analyseur d'images permet d'obtenir une précision très satisfaisante.

Contrairement aux observations faites par PETTIPHer et al. (1980) puis par SuHREN et HeEschen (1984), nous avons constaté, pour certaines lames, une diminution de l'intensité, voire une disparition complète de la coloration des bactéries après un stockage prolongé des lames à $4^{\circ} \mathrm{C}$. 


\section{Remerciements}

Nous remercions la Société Foss Electric France pour le prêt de l'appareil et pour l'aide technique qu'elle nous a apportée.

\section{Références bibliographiques}

ANONYME, 1972. Standard methods for the examination of dairy products. American Public Health Association, 13th ed., New York.

ANoNYME, 1981. Lait liquide. Dénombrement des micro-organismes (technique par comptage des colonies à $30^{\circ} \mathrm{C}$ ). Norme provisoire F.I.L. 100.

BECK C.G., HeHIR A.F., 1982. The rapid fluorescence method for counting bacteria in milk. Aust. J. Dairy Technol., 37, 66-67.

Bossuyt R., 1982. Techniques modernes d'appréciation de la qualité bactériologique du lait. Tech. Lait., (969), 9-19,

BREED R.S., BREW J.D., 1916. Counting bacteria by means of the microscope. Technical Bulletin $n^{\circ} 49$, New York Agricultural Experiment Station.

Grappin R., 1976. Guide pour l'évaluation des méthodes d'analyses de routine. Lait, 52, 608-621.

GrapPin R., Jeunet R., 1974. Progrès récents dans la mécanisation d'une méthode simplifiée de numération des germes totaux du lait. 19e Cong. Int. Lait., B 8, 576-577.

Grappin R., Dasen A., Favennec P., 1985. Numération des bactéries du lait cru à l'aide du Bacto-Scan. Lait, 65, 123-147.

HobBIE J.E., DALEY R.J., JASPER S., 1977. Use of nuclepore filters for counting bacteria by fluorescence microscopy. Appl. Environ. Microbiol., 33, 1225-1228.

HunTER A.C., Mac CoRouodale R.M., 1983. Evaluation of the direct epifluorescent filter technique for assessing the hygienic condition of milking equipment. J. Dairy Res., 50, 9-16.

JONES C.G., SIMON B.M., 1975. An investigation of errors in direct counts of aquatic bacteria by epifluorescence microscopy, with reference to a new method of dyeing membrane filters. J. Appl. Bacteriol., 39, 317-329.

Kirchman D., Sigda J., Kapuscinski R., Mitchell R., 1982. Statiscal analysis of the direct count method for enumerating bacteria. Appl. Environ. Microbiol., 44, 376-382.

O'CONNOR F., 1984. Rapid test methods for assessing microbiological quality of milk. Aust. J. Dairy Technol., 39, 61-65.

O'TOOLE D.K., 1983. A review : methods for the direct and indirect assessment of the bacterial content of milk. J. Appt. Bacteriol., 55, 187-201.

PETTIPHER G.L., 1981. Rapid methods for assessing bacterial numbers in milk. Dairy Ind. Int., 46, 15-23.

PETTIPHER G.L., RODRIGUES U.M., 1981. Rapid enumeration of bacteria in heat-treated milk and milk products using a membrane filtration - epifluorescent microscopy technique. J. Appl. Bacteriol., 50, 157-166.

PETTIPHER G.L., RoDRIGUES U.M., 1982 a. Rapid enumeration of microorganisms in foods by the direct epifluorescent filter technique. Appl. Environ. Microbiol., 44, 809-813.

PETTIPHER G.L., RODRIGUeS U.M., 1982 b. Semi-automated counting of bacteria and somatic cells in milk using epifluorescence microscopy and television image analysis. J. Appl. Bacteriol., 53, 323-329.

Pettipher G.L., Mansell R., Mac Kinnon C.H., Cousins C.M., 1980. Rapid membrane filtration - epifluorescent microscopy technique for direct enumeration of bacteria in raw milk. Appl. Environ. Microbiol., 39, 423-429. 
PetitPher G.L., Fulford R.J., MabittT L.A., 1983. Collaborative trial of the direct epifluorescent filter technique (DEFT), a rapid method for counting bacteria in milk. J. Appl. Bacteriol., 54, 177-182.

Piton C., RichaRd J., 1983. Influence de l'agitation des échantillons de lait cru sur les résultats de dénombrement de trois groupes microbiens d'intérêt technologique. Lait, 63, 405-415.

RICHARD J., 1980. Influence de l'agitation du lait cru sur les résultats de dénombrement de sa flore totale à l'aide d'une anse calibrée. Lait, 60, 211-225.

RICHARD J., 1981. Influence de diverses méthodes de nettoyage des machines à traire sur la "qualité de conservation " du lait cru à basse température. Lait, 61, 354-369.

Richard J., Krebs C., Walter J. Grappin R., 1981. Comparaison de divers modes d'agitation des échantillons de lait cru en vue de la numération de sa flore totale suivant la méthode Thompson. Lait, 61, 250-260.

SUHREN G., HEESCHEN W., 1984. Untersuchungen zur Keimzahlbestimmung in Rohmilch mit der direkten Epifluoreszenz - Filter - Technik DEFT. Kiel. Milchwirtsch. Forsch. Ber., 36, 87-136.

Von BeRtalanfFy L., BICKIS I., 1956. Identification of cytoplasmic basophilia (Ribonucleic Acid) by fluorescence microscopy. J. Histochem. Cytochem., 4, 481-493. 